\title{
Caracterização de Acetato de Celulose Obtido a partir do Bagaço de Cana-de-Açúcar por ${ }^{1} \mathrm{H}-\mathrm{RMN}$
}

\author{
Daniel A. Cerqueira \\ Instituto de Ciências Ambientais e Desenvolvimento Sustentável, UFBA
}

Guimes Rodrigues Filho Instituto de Química, UFU

Rui de A. Carvalho Departamento de Bioquímica e CNC, Universidade de Coimbra, Portugal

Artur J. M. Valente Departamento de Química, Universidade de Coimbra, Portugal

\begin{abstract}
Resumo: A celulose do bagaço de cana-de-açúcar foi usada para sintetizar acetato de celulose com diferentes graus de substituição, os quais foram caracterizados por ${ }^{1} \mathrm{H}-\mathrm{RMN}$ através da relação entre as áreas dos picos dos átomos de hidrogênio presentes nos grupos acetato $\left(-(\mathrm{C}=\mathrm{O}) \mathrm{OCH}_{3}\right)$ e os picos dos hidrogênios ligados aos átomos de carbono dos anéis glicosídicos. A supressão de alguns sinais foi feita para remover sinais de ressonância da água residual nos materiais e também para remover sinais de impurezas no triacetato de celulose. Um método de deconvolução para o cálculo computacional do grau de substituição foi proposto. Os graus de substituição das amostras de acetato de celulose foram 2,94 e 2,60, o que está de acordo com os resultados por determinação química através de uma titulação ácido base.
\end{abstract}

Palavras-chave: Acetato de celulose, bagaço de cana-de-açúcar, grau de substituição, ${ }^{1} H$-RMN, deconvolução.

\section{'H-NMR Characterization of Cellulose Acetate Obtained from Sugarcane Bagasse}

\begin{abstract}
Cellulose from sugarcane bagasse was used for synthesizing cellulose acetate with different degrees of substitution, which were characterized by ${ }^{1} \mathrm{H}-\mathrm{NMR}$ through the relationship between the peak areas of the hydrogen atoms of the acetate groups $\left(-(\mathrm{C}=\mathrm{O}) \mathrm{OCH}_{3}\right)$ and the peaks of the hydrogen bonded to the carbon atoms of the glucosidic rings. Suppression of some signals was carried out in order to remove the residual water resonance in the materials and those related to impurities in cellulose triacetate as well. A deconvolution method for the computation of the degree of substitution of acetylation is proposed. The degrees of substitution for the cellulose samples were 2.94 and 2.60, in good agreement with those obtained by chemical determination through an acid-base titration.
\end{abstract}

Keywords: Cellulose acetate, sugarcane bagasse, degree of substitution, ${ }^{1} \mathrm{H}-\mathrm{NMR}$, deconvolution.

\section{Introdução}

De acordo com dados de 2005 da Organização das Nações Unidas para Agricultura e Alimentação (FAO), o Brasil é o maior produtor mundial de cana-de-açúcar e a sua produção é maior do que a dos quatro maiores produtores seguintes (Índia, China, Tailândia e Paquistão) juntos ${ }^{[1]}$. De acordo com o Instituto Brasileiro de Geografia e Estatística (IBGE), no ano de 2007 foram produzidas 548 milhões de $\mathrm{t}$ de cana-de-açúcar e em 2008 a produção estimada foi de 653 milhões de t, um aumento de aproximadamente $19 \%^{[1]}$. Este aumento deve-se essencialmente ao crescimento da procura de álcool combustível, tanto para o mercado interno como para a exportação, que vem incentivando a expansão da plantação de cana-de-açúcar e o surgimento de novas usinas. Somente no Triângulo Mineiro, estão instaladas 17 das 30 Usinas do Estado de Minas Gerais. Atualmente, o Brasil produz $17 \times 10^{9} \mathrm{~L}$ de álcool por ano, com uma área plantada de 3 milhões de ha de cana-de-açúcar ${ }^{[1]}$.

A quantidade de bagaço de cana-de-açúcar seco oriunda dessa atividade corresponde a $280 \mathrm{~kg}$ para cada tonelada de cana-de-açúcar processada. Atualmente, o bagaço de cana de açúcar é em sua maioria queimado para gerar energia para as usinas ${ }^{[2]}$, mas uma quantidade considerável é ainda desperdiçada, e por essa razão, vários artigos têm sido produzidos buscando alternativas para sua reciclagem. Por

Autor para correspondência: Daniel A. Cerqueira, Instituto de Ciências Ambientais e Desenvolvimento Sustentável, Universidade Federal da Bahia, Rua Professor José Seabra S/N, CEP: 47805-100, Barreiras, BA, Brasil. E-mail: daniel.cerqueira@ufba.br 
exemplo, a utilização da celulose para a produção de álcool pelo processo de hidrólise enzimática e hidrólise ácida ${ }^{[3-5]}$, produção de compósitos ${ }^{[6]}$ e derivados celulósicos ${ }^{[7-10]}$.

$\mathrm{O}$ acetato de celulose (AC), Figura 1, é um dos derivados da celulose com maior importância comercial, principalmente devido às seguintes propriedades: é um polímero neutro, tem a capacidade de formação de filmes transparentes e tem um baixo custo. O AC pode ser usado em processos de separação por membranas, tais como hemodiálise, nanofiltração e osmose inversa $^{[11]}$, matrizes para libertação controlada de fármacos ${ }^{[12]}$, sensores e proteção de filmes ópticos ${ }^{[13,14]}$, separação de gases $^{[15]}$ e preparação de filmes de alumina ${ }^{[16]}$. Outro interessante campo de aplicação do AC é a sua utilização como matriz para incorporação de, e.g., polímeros condutores $^{[17]}$ e macrociclos ${ }^{[18-20]}$. Além disso, blendas de AC e polianilina têm sido usadas para o desenvolvimento de dispositivos eletrônicos e eletrocrômicos ${ }^{[21-23]}$, e eletrodos seletivos $^{[24]}$.

Os derivados acetilados da celulose podem ser produzidos por dois tipos diferentes de reações de acetilação: homogênea e heterogênea ${ }^{[25]}$. Ambas as reações ocorrem geralmente através do uso de ácido acético como solvente, anidrido acético como agente acetilante, e ácidos sulfúrico e perclórico como catalisadores. $\mathrm{O}$ acetato de celulose é, assim, produzido pela substituição dos grupos hidroxila das unidades de glicose por grupos acetila. Como consequência pode-se obter materiais com diferentes graus de substituição (GS) (sendo o grau de substituição o número médio de grupos acetila que substituem as hidroxilas por unidade glicosídica), o qual pode variar de zero (para a celulose) a 3 (para um material trisubstituído). O GS é um parâmetro de extrema importância, pois afeta a cristalinidade do polímero ${ }^{[26,27]}$, o potencial de biodegradabilidade e a solubilidade em diferentes solventes, entre outras proprieades. Por exemplo, celulose $(G S=0)$ é insolúvel na maioria dos solventes $(\mathrm{S})$, mas ao se aumentar o GS do acetato de celulose, a solubilidade se altera $(G S \approx 1$, $\mathrm{S}$ : água; $\mathrm{GS} \approx 2, \mathrm{~S}$ : tetraidrofurano ou acetona; $\mathrm{GS} \approx 3$, S: diclorometano ou clorofórmio ${ }^{[27]}$. Assim, a determinação do GS do acetato de celulose é importante para que se defina sua utilização.

Várias são as técnicas experimentais comumente para a determinação do GS: por via química (ver seção experimental) ou por técnicas instrumentais de análise. Dentre estas últimas, as mais usadas são a cromatografia gáslíquido, espectrometria de massa, cromatografia líquida de

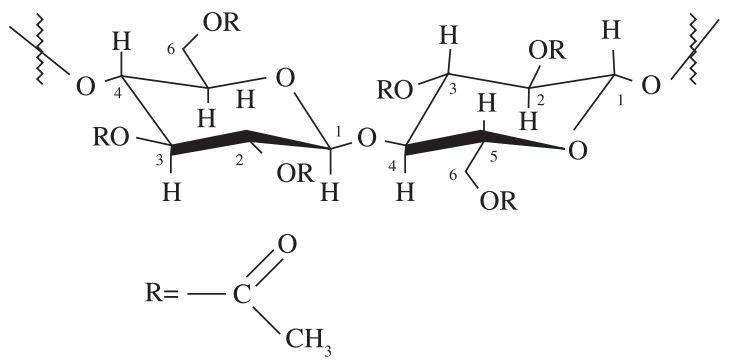

Figura 1. Estrutura do acetato de celulose. alta eficiência, espectroscopia e infravermelho, e ressonância magnética nuclear de prótons ( $\left.{ }^{1} \mathrm{H}-\mathrm{RMN}\right)$ e de carbono $\left({ }^{13} \mathrm{C}-\mathrm{RMN}\right){ }^{[27]}$.

Tanto nas análises de ${ }^{1} \mathrm{H}-\mathrm{RMN}$ quanto de ${ }^{13} \mathrm{C}-\mathrm{RMN}$ as amostras são geralmente dissolvidas em dimetil sulfóxido deuterado (DMSO-d6) o qual pode dissolver amostras de acetato de celulose em uma ampla faixa de GS. Embora ambas as técnicas sejam eficientes na caracterização de amostras de alto GS, à medida em que há uma menor substituição da amostra os espectros tornam-se mais complexos devido à sobreposição de sinais. As alternativas para resolver esse problema utilizam as hidroxilas não substituídas para a produção de derivados a partir de reações como trifluoracetilação, nitrobenzoilação, etilcarbamilação, fenilcarbamilação, trimetilsililação e conversão com isocianoacetato de etila, ou ainda a peracetilação, utilizando reagentes deuterados. Os derivados resultantes dessas reações levam a um espectro de ressonância mais bem definido e permitem o cálculo do GS com grande precisão quando comparados com valores obtidos pela via química ${ }^{[27]}$. No entanto, esta estratégia dificulta bastante a disseminação e a rapidez da análise.

Nesse trabalho, a determinação do GS do acetato de celulose obtido a partir do bagaço de cana-de-açúcar ${ }^{[1,28]}$ foi estudada por ressonância magnética nuclear de prótons ( $\left.{ }^{1} \mathrm{H}-\mathrm{RMN}\right)$. Os espectros de ${ }^{1} \mathrm{H}-\mathrm{RMN}$ passaram por um processo de deconvolução matemática para a eliminação de picos referentes a impurezas do material, sendo que dada a resolução dos espectros não foi necessária a derivatização das amostras de acetato de celulose. O modelo de deconvolução agora apresentado permitirá a fácil e correta determinação do GS com base em espectroscopia de ${ }^{1} \mathrm{H}-\mathrm{RMN}$. Os resultados obtidos por esta técnica e pelo método de deconvolução proposta apresentam uma boa concordância com os obtidos através da determinação por via química.

\section{Experimental}

\section{Materiais}

Bagaço de cana-de-açúcar da safra de 2007 foi fornecido pela Usina Caeté, de Delta, Minas Gerais, Brasil, purificado de acordo o método descrito por Rodrigues Filho et al. ${ }^{[29]}$. Hidróxido de sódio e dimetilsulfoxido deuterado (DMSO-d6) foram fornecidos pela Sigma-Aldrich. Todos os outros reagentes e solventes foram fornecidos pela Panreac. Todos os reagentes e solventes foram utilizados como adquiridos.

\section{Acetilação da celulose do bagaço de cana-de-açúcar}

O triacetato de celulose foi produzido de acordo com Cerqueira et al. ${ }^{[28]}$, cujo procedimento é descrito a seguir:

Uma mistura composta por $1 \mathrm{~g}$ de bagaço purificado e $25 \mathrm{~mL}$ de ácido acético foi agitada durante 30 minutos. Então, uma solução composta por $0,08 \mathrm{~mL} \mathrm{H}_{2} \mathrm{SO}_{4}$ e $9 \mathrm{~mL}$ de ácido acético foi adicionada ao sistema, o qual foi agitado durante 
25 minutos. A mistura foi filtrada e $32 \mathrm{~mL}$ de anidrido acético foram adicionados ao filtrado. Essa solução foi recolocada no recipiente contendo bagaço e agitada durante 30 minutos. Após esse período, a mistura ficou em repouso por 14 horas a $28{ }^{\circ} \mathrm{C}$. A mistura foi filtrada para remover as partículas não dissolvidas. Água foi adicionada ao filtrado para parar a reação e precipitar o triacetato de celulose (TAC), o qual foi lavado com água destilada para remover o ácido acético, e então seco à temperatura ambiente.

Para produzir o diacetato de celulose (DAC), utilizou-se uma variação do procedimento de produção do TAC. Antes da etapa de adição de água para parar a reação adicionouse uma mistura contendo $10 \mathrm{~mL}$ de ácido acético, $10 \mathrm{~mL}$ de água e $0,75 \mathrm{~mL}$ de ácido sulfúrico, lentamente, ao meio reacional. $\mathrm{O}$ frasco foi imerso em um banho de água a $80{ }^{\circ} \mathrm{C}$ por 10 minutos, e após esse período, adicionou-se água à mistura para precipitar o diacetato de celulose, o qual foi filtrado e lavado para remover o excesso de ácido acético, e então seco à temperatura ambiente.

\section{Espectrometria de ${ }^{1} H-R M N$}

Os espectros de ${ }^{1} \mathrm{H}-\mathrm{RMN}$ foram obtidos a partir de soluções de diacetato ou triacetato de celulose obtidos a partir da celulose do bagaço de cana-de-açúcar dissolvidos em dimetil sulfóxido deuterado (DMSO-d6) na proporção de $20 \mathrm{mg} \cdot \mathrm{g}^{-1}$ (polímero/solvente). Os espectros foram adquiridos à temperatura de $50{ }^{\circ} \mathrm{C}$ num espectrômetro de RMN Varian Unity 500 usando uma sonda de $5 \mathrm{~mm}$. Parâmetros de aquisição típicos incluíram um pulso de radiofrequências de $45^{\circ}$, um tempo de repetição interpulsos de 8 segundos e 32000 pontos cobrindo uma largura espectral de $7200 \mathrm{~Hz}$. Antes de proceder à transformação de Fourier do espectro, o decaimento livre induzido ('FID') foi multiplicado por uma função de apodização Lorentziana de $0,5 \mathrm{~Hz}$. Os deslocamentos químicos foram calculados relativos ao sinal obtido para o DMSO-d6 em 2,49 ppm.

\section{Determinação do grau de substituição por via química}

Adicionaram-se $5 \mathrm{~mL}$ de hidróxido de sódio $0,25 \mathrm{~mol} . \mathrm{L}^{-1}$ e $5 \mathrm{~mL}$ de etanol a $0,1 \mathrm{~g}$ de acetato de celulose. Após 24 horas adicionaram-se $10 \mathrm{~mL}$ de ácido clorídrico $\left(0,25 \mathrm{~mol} . \mathrm{L}^{-1}\right)$ e deixou-se em repouso por mais 30 minutos. Em seguida a solução foi retrotitulada com hidróxido de sódio padronizado com biftalato de potássio, utilizando-se fenolftaleína como indicador.

A determinação do grau de substituição (GS) por via química baseia-se na determinação da porcentagem $(\% \mathrm{~m} / \mathrm{m})$ de grupos acetila (GA) que foram substituídos na cadeia celulósica. Ou seja, o GS é determinado a partir do valor de GA pela Equação $1^{[30] \text { : }}$

$$
G S=\frac{162 \times G A}{43 \times 100-42 \times G A} \times 100
$$

em que o GA é calculado a partir da Equação 2.

$$
G A=\frac{\left[V_{b} \mu_{b}-V_{a} \mu_{a}\right] \times 43 \times 100}{m_{a c}}
$$

onde $\mathrm{V}_{\mathrm{b}}$ é a soma do hidróxido de sódio adicionado no procedimento e gasto na titulação, $\mathrm{V}_{\mathrm{a}}$ é o volume de ácido clorídrico adicionado, $\mu_{\mathrm{a}}$ e $\mu_{\mathrm{b}}$ são a molaridade das soluções de ácido clorídrico e hidróxido de sódio, respectivamente, e $\mathrm{m}_{\mathrm{ac}}$ é a massa de acetato de sódio utilizada.

\section{Resultados e Discussão}

O procedimento de purificação do bagaço utilizado nesse trabalho ${ }^{[29]}$ leva à produção de um material com $75 \%$ de celulose, $4 \%$ de lignina e o restante de hemicelulose ${ }^{[28]}$. Apesar disso, a reação de acetilação ocorre sem problemas, produzindo cerca de 1,5 g de triacetato de celulose para cada grama de bagaço purificado. Tanto para o triacetato de celulose quanto para o diacetato de celulose, não há evidência da presença de lignina, hemicelulose ou seus derivados nos espectros de ${ }^{1} \mathrm{H}-\mathrm{RMN}$, mostrados na Figura 2. Nesses espectros, observam-se entre 3,5 e 5,3 ppm os picos relacionados aos átomos de hidrogênio ligados aos carbonos dos grupos glicosídicos, onde o índice representa o átomo de carbono ao qual o átomo de hidrogênio está ligado. Entre 1,75 e 2,20 ppm surgem os picos relacionados com os átomos de hidrogênio metílicos nos grupos acetato.

$\mathrm{O}$ grau de substituição por ${ }^{1} \mathrm{H}-\mathrm{RMN}$ é calculado pela relação entre as áreas atribuídas aos hidrogênios dos grupos metílicos $\left(\mathrm{A}_{\mathrm{CH}_{3}}\right)$ e aos hidrogênios dos anéis glicosídicos $\left(\mathrm{A}_{\text {glic }}\right)$, de acordo com a Equação 3.

$$
G S=\frac{7}{3} \times \frac{A_{\mathrm{CH}_{3}}}{A_{\text {glic. }}}
$$

Entretanto, em aproximadamente $1,90 \mathrm{ppm}$ no espectro de ${ }^{1} \mathrm{H}-\mathrm{RMN}$ do triacetato de celulose observa-se um sinal provavelmente relacionado a alguma impureza residual do processo de acetilação. Tal sinal não é observado no espectro do diacetato de celulose. Recorreu-se então a uma deconvolução para a retirada deste sinal. A deconvolução foi

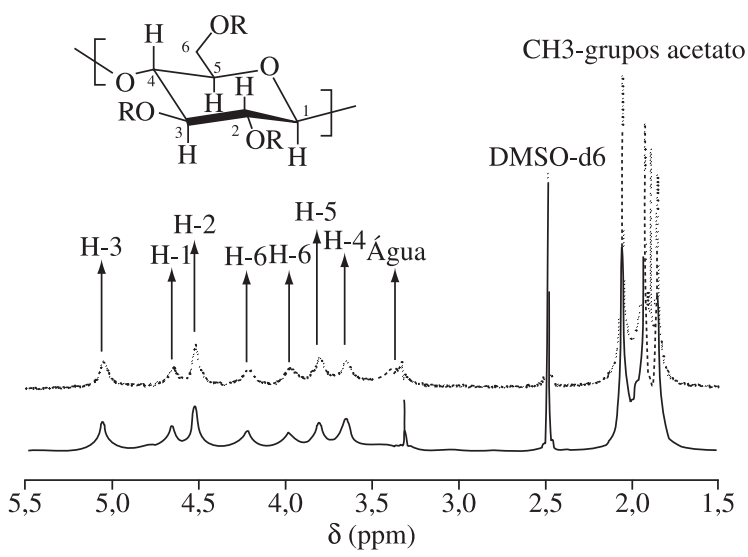

Figura 2. Espectros de ${ }^{1} \mathrm{H}-\mathrm{RMN}$ de amostras de triacetato (pontilhado) e diacetato (contínuo) de celulose 
efetuada utilizando-se a função Lorentziana no programa OriginPro 7.5G, mostrada na Equação 4.

$$
y=y_{0}+\frac{2 A}{\pi} \frac{w}{4\left(x-x_{c}\right)^{2}+w^{2}}
$$

Para a deconvolução dos sinais, tomou-se como modelo o espectro do diacetato de celulose (Figura 3a). Inicialmente atribuiu-se os 3 sinais principais em 2,06, 1,93 e 1,86 ppm (Figura 3b). Observou-se ainda um alargamento na base do sinal em 1,93 ppm, relativo aos acoplamentos com os outros sinais. Assim, para que se obtivesse um melhor ajuste, foi adicionada uma nova curva nesta região, deixando que o programa ajustasse a melhor posição para a mesma. Desta forma, o valor para o máximo desta curva ocorreu em 1,96 ppm (Figura 3c). O ajuste final é mostrado na Figura 3d.

O mesmo modelo foi seguido para a deconvolução da região dos grupos metila no espectro de ${ }^{1} \mathrm{H}-\mathrm{RMN}$ do triacetato de celulose, como mostrado na Figura 4. Os sinais em 2,06, 1,96, 1,93 e 1,86 ppm, existentes no espectro do diacetato de celulose foram fixados (Figura $4 \mathrm{~b}$ ), adicionando-se uma curva extra na região de 1,90 ppm (Figura 4c), referente ao sinal a ser eliminado da curva. O resultado da deconvolução, já com a eliminação do sinal em 1,90 ppm é mostrado na Figura 4d.

As áreas referentes aos sinais dos grupos metila nos espectros de ${ }^{1} \mathrm{H}$-RMN do diacetato de celulose e triacetato de celulose são apresentadas na Tabela 1 .

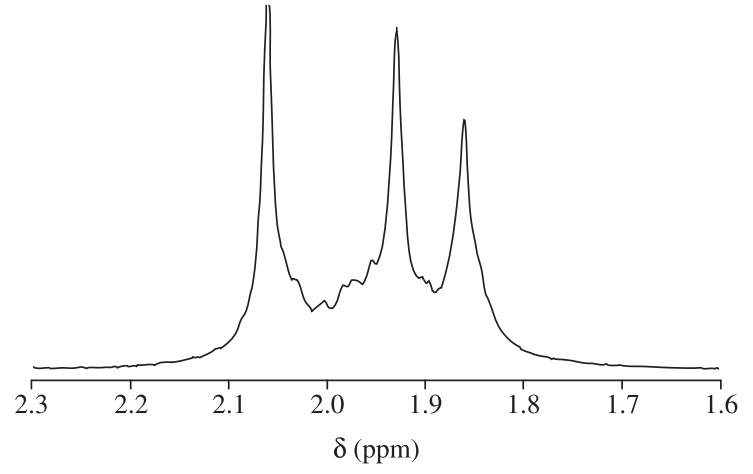

(a)

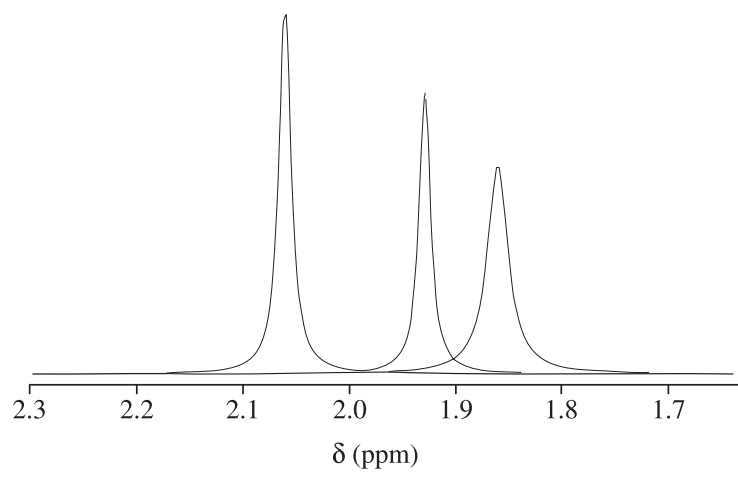

(b)
A região correspondente aos sinais dos prótons dos grupos glicosídicos também foi deconvoluída com o propósito de remover a interferência do sinal de água, em 3,31 ppm. A sequência de deconvolução foi semelhante à utilizada anteriormente, iniciando-se com a atribuição dos sinais principais: 5,06 (H-3); 4,66 (H-1); 4,52 (H-2); 4,22 (H-6); 3,99 (H-6); 3,81 (H-5), 3,66 (H-4) e 3,31 ppm $\left(\mathrm{H}_{2} \mathrm{O}\right)$. Em seguida, adicionou-se mais dois sinais relativos à interação entre os sinais principais em 4,63 e 3,44 ppm. A escolha destes dois sinais, apesar de aleatória, é coerente quando se leva em consideração que os sinais referentes aos prótons ligados aos átomos de carbono 3,1 e 2 no anel glicosídico (com maior influência do sinal em 4,63 ppm) estão geometricamente opostos aos prótons ligados aos átomos de carbono 6, 5 e 4 (com maior influência do sinal em 3,44), como pode ser visto na estrutura mostrada na Figura 1. As sequências de deconvolução para o diacetato de celulose e para o triacetato de celulose são mostradas nas Figuras 5 e 6 , respectivamente, e os valores calculados para as áreas são mostrados na Tabela 1 .

A partir desses dados de deconvolução, o GS das amostras foi determinado utilizando-se a Equação 3, obtendo-se o valor de 2,60 $( \pm 0,60)$ e 2,88 $( \pm 0,05)$ para o DAC e TAC, respectivamente. Esses valores estão de acordo com os obtidos por titulação ácido base: $2,65( \pm 0,04)$ e $2,94( \pm 0,06)$.

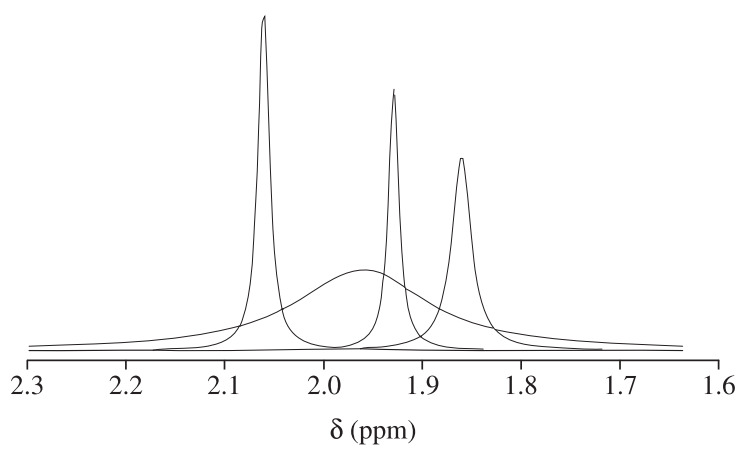

(c)

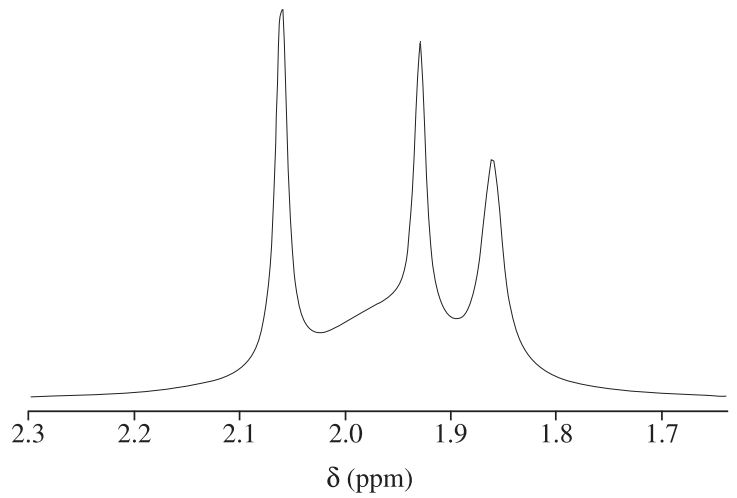

(d)

Figura 3. Sequência de deconvolução para a região correspondente aos sinais do grupo metila no espectro de ${ }^{1} \mathrm{H}-\mathrm{RMN}$ do diacetato de celulose. a) espectro original; b) adição dos sinais principais; c) adição do sinal secundário; d) somatório das curvas de deconvolução. 


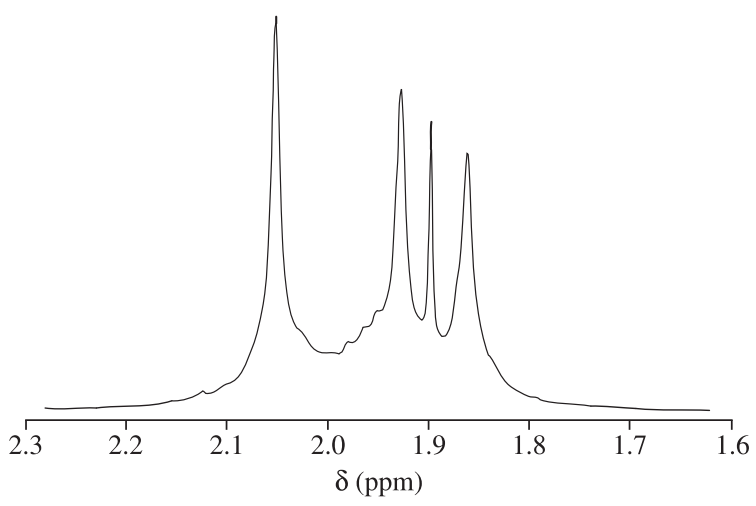

(a)

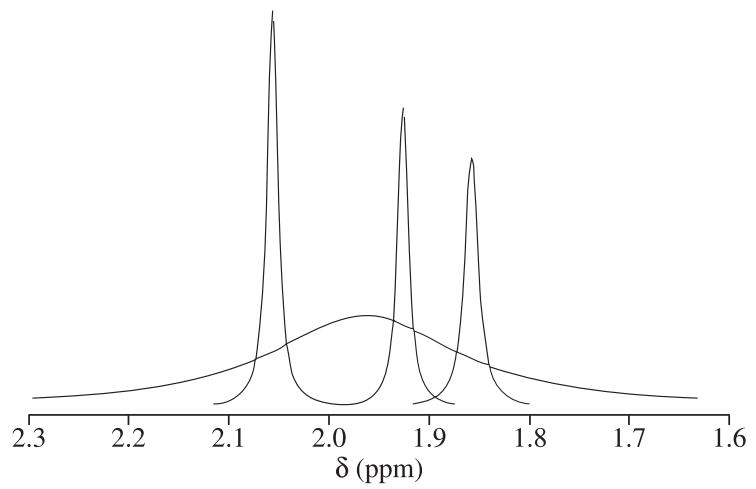

(b)

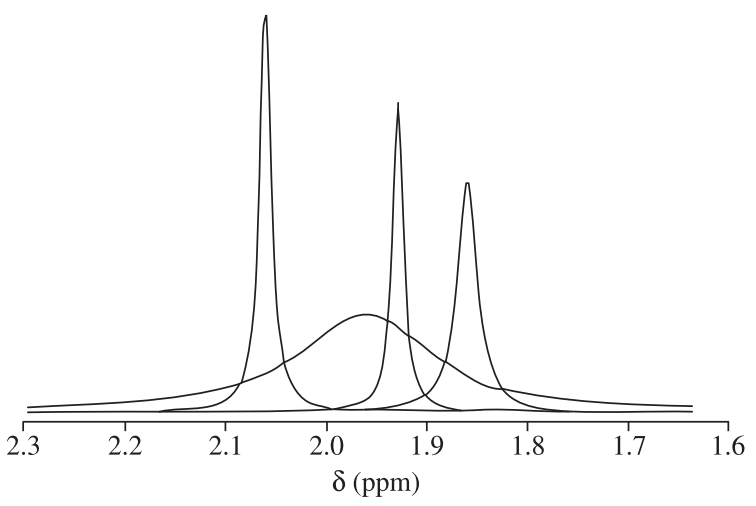

(c)

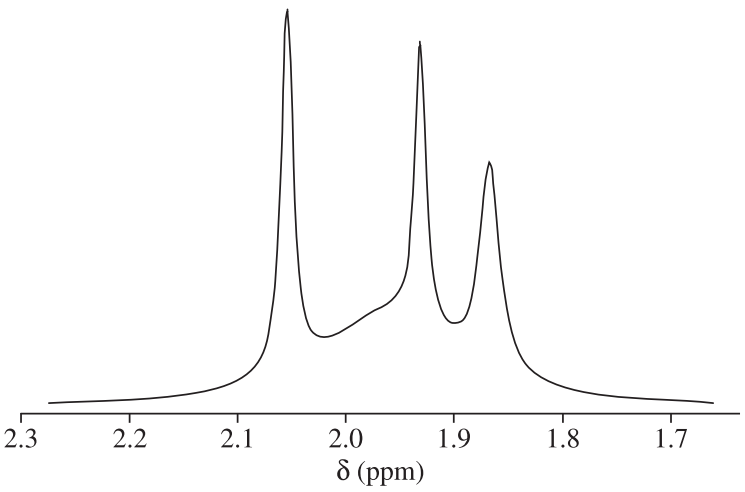

(d)

Figura 4. Sequência de deconvolução para a região correspondente aos sinais do grupo metila no espectro de ${ }^{1} \mathrm{H}-\mathrm{RMN}$ do triacetato de celulose. a) espectro original; b) adição dos sinais de acordo com o espectro do diacetato de celulose; c) adição do sinal relativo ao interferente; d) somatório das curvas de deconvolução.

Tabela 1. Áreas calculadas a partir das deconvoluções dos espectros de ${ }^{1} \mathrm{H}-\mathrm{R} M N$ de diacetato de celulose e triacetato de celulose.

\begin{tabular}{|c|c|c|c|}
\hline & & Diacetato de Celulose & Triacetato de celulose \\
\hline & Deslocamento (ppm) & Área $\pm \mathbf{s}^{*}(\mathbf{u} . \mathbf{a})$. & Área $\pm \mathbf{s}^{*}$ (u.a.) \\
\hline \multirow[t]{5}{*}{ Prótons dos grupos Metílicos } & 2,06 & $41233 \pm 432$ & $48899 \pm 1190$ \\
\hline & 1,93 & $34676 \pm 574$ & $72076 \pm 3370$ \\
\hline & 1,86 & $39843 \pm 771$ & $61098 \pm 500$ \\
\hline & 1,96 & $132268 \pm 4286$ & $56084 \pm 719$ \\
\hline & - & $\mathrm{A}_{\mathrm{CH}_{3}}=248020 \pm 4414$ & $\mathrm{~A}_{\mathrm{CH}_{3}}=238158 \pm 3680$ \\
\hline \multirow[t]{10}{*}{ Prótons do anel glicosídico } & 5,06 & $20670 \pm 154$ & $20555 \pm 121$ \\
\hline & 4,66 & $14867 \pm 287$ & $12333 \pm 258$ \\
\hline & 4,52 & $21303 \pm 157$ & $20970 \pm 182$ \\
\hline & 4,22 & $17079 \pm 196$ & $13343 \pm 158$ \\
\hline & 3,99 & $17573 \pm 187$ & $12321 \pm 245$ \\
\hline & 3,81 & $20966 \pm 163$ & $17282 \pm 317$ \\
\hline & 3,66 & $25437 \pm 187$ & $19357 \pm 240$ \\
\hline & 4,63 & $53339 \pm 2490$ & $26602 \pm 710$ \\
\hline & 3,44 & $31356 \pm 1040$ & $50084 \pm 1516$ \\
\hline & & $A_{\text {glic }}=222592 \pm 2748$ & $\mathrm{~A}_{\text {glic }}=192847 \pm 1778$ \\
\hline
\end{tabular}

* s é o desvio padrão associado ao cálculo do valor da área. 


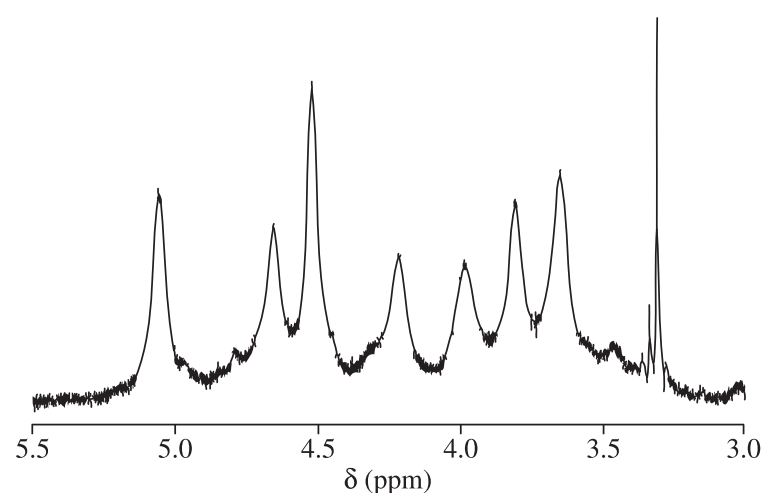

(a)

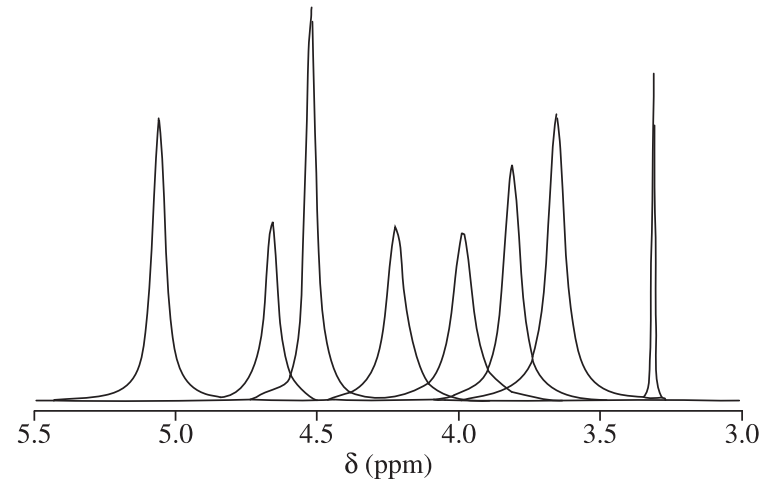

(b)

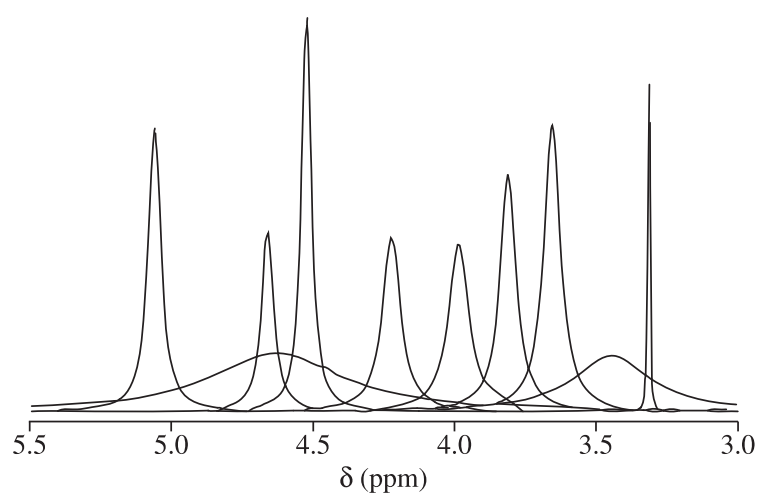

(c)

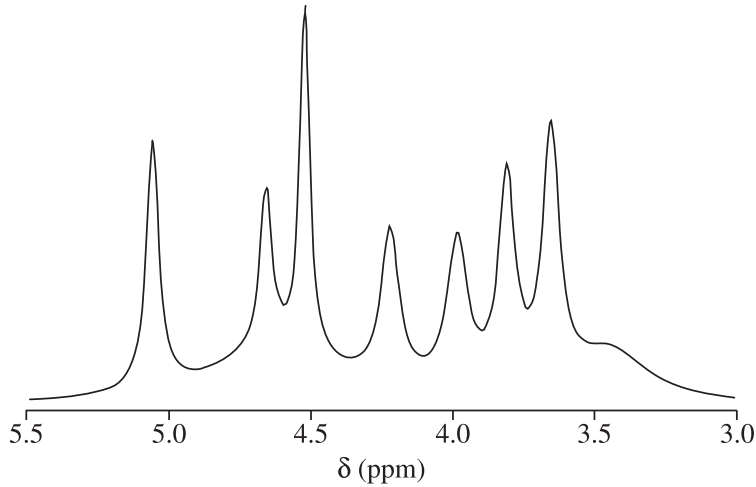

(d)

Figura 5. Sequência de deconvolução para a região correspondente aos sinais dos prótons ligados ao grupo glicosídico no espectro de ${ }^{1} \mathrm{H}-\mathrm{RMN}$ do diacetato de celulose. a) espectro original; b) adição dos sinais principais; c) adição dos sinais relativos aos acoplamentos entre os sinais principais; d) somatório das curvas de deconvolução, excluindo-se o sinal da água.

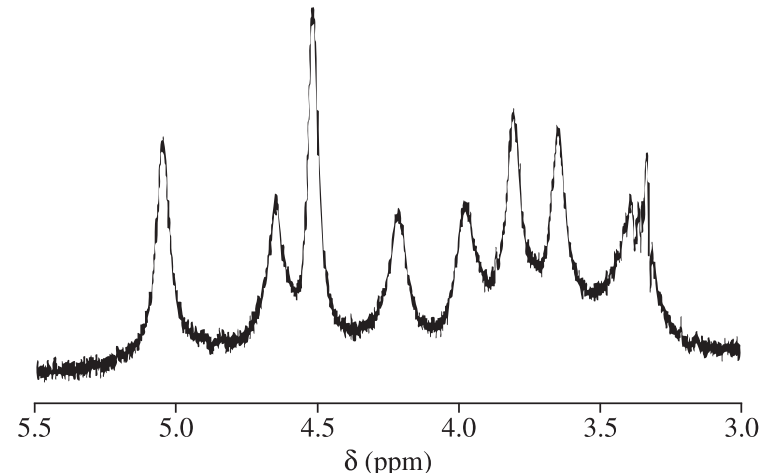

(a)

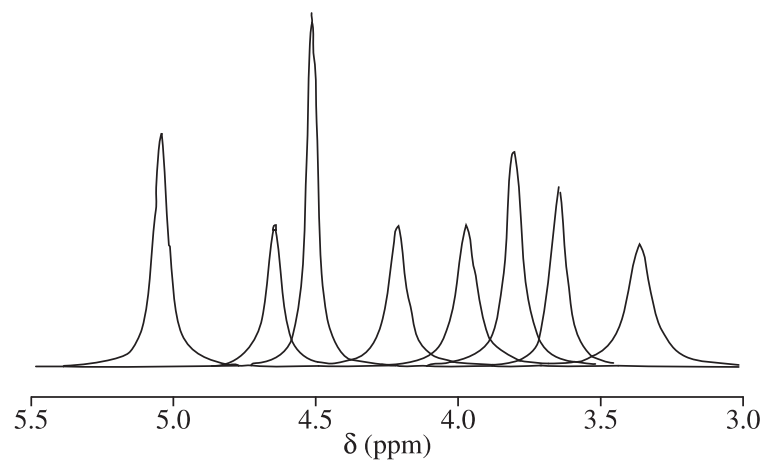

(b)

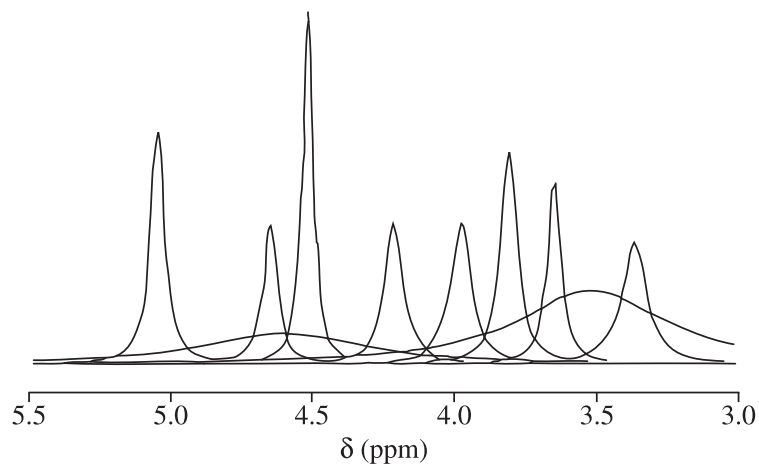

(c)

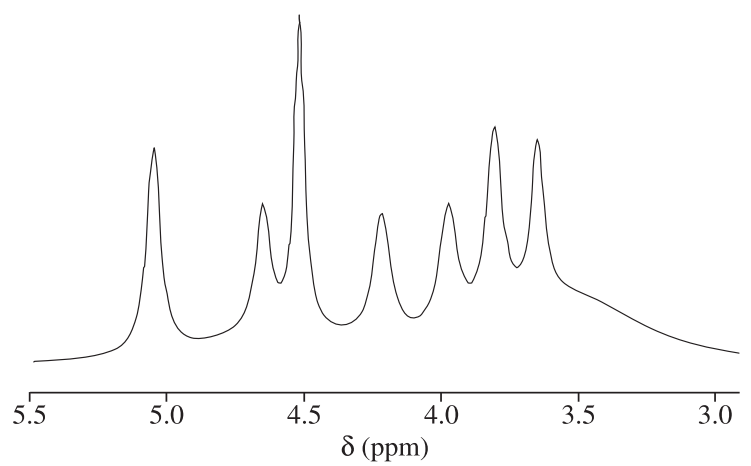

(d)

Figura 6. Sequência de deconvolução para a região correspondente aos sinais dos prótons ligados ao grupo glicosídico no espectro de ${ }^{1} \mathrm{H}-\mathrm{RMN}$ do triacetato de celulose. a) espectro original; b) adição dos sinais principais; c) adição dos sinais relativos aos acoplamentos entre os sinais principais; d) somatório das curvas de deconvolução, excluindo-se o sinal da água. 


\section{Conclusão}

Os Graus de Substituição (GS) de amostras de acetato de celulose foram determinados a partir da deconvolução dos espectros de ${ }^{1} \mathrm{H}-\mathrm{RMN}$, e os valores obtidos $(2,60$ para o diacetato de celulose e 2,88 para o triacetato de celulose) estão em boa concordância com os valores obtidos por titrimetria $(2,65$ e 2,94 para o di e triacetato de celulose, respectivamente). O método de deconvolução descrito neste artigo associa simplicidade à determinação rigorosa dos graus de substituição dos acetatos de celulose.

\section{Agradecimentos}

Cerqueira agradece à CAPES por sua bolsa de doutorado e bolsa de sanduíche (BEX 0368/07-5). Os autores agradecem ainda à FAPEMIG.

\section{Referências}

1. Cerqueira, D. A. - "Síntese e caracterização de misturas poliméricas contendo acetato de celulose: aproveitamento de resíduos da cana-de-açúcar", $\mathrm{PhD}$. Thesis, Universidade Federal de Uberlândia, Uberlândia (2009).

2. Gamez, A.; Gonzalez-Cabriales, J. J.; Ramirez, J. A.; Garrote, G. \& Vazquez M. - J. Food Eng., 74, p. 78 (2006).

3. Banerjee, R. \& Pandey, A. - Int. Sugar J., 104, p. 64 (2002).

4. Martin, C.; Klinke, H. B. \& Thomsen, A. B. Enzym Microb. Tech., 40, p. 426 (2007).

5. Sarrouh, B. F.; Silva, S. S.; Santos, D. T. \& Converti, A. - Chem. Eng. Technol. 30, p. 270 (2007).

6. Ramaraj, B. - J. Appl. Polym. Sci. 103, p. 3827 (2007).

7. Liu, C. F.; Sun, R. C.; Zhang, A. P. \& Ren, J. L. Carbohyd. Polym. 68, p. 17 (2007).

8. Pasquini, D.; Belgacem, M. N.; Gandini, A. \& Curvelo, A. A. D. - J. Colloid Interface Sci., 295, p. 79 (2006).

9. Viera, R. G. P.; Rodrigues Filho, G.; Assunção, R. M. N.; Meireles, C. S.; Vieira, J. G. \& Oliveira, G. S. Carbohyd. Polym. 67, p. 182 (2007).

10. Rodrigues Filho, G.; Assunção, R. M. N.; Vieira, J. G.; Meireles, C. S.; Cerqueira, D. A.; Barud, H. D.; Ribeiro, S. J. L. \& Messaddeq, Y. - Polym. Degrad. Stab. 92, p. 205 (2007).

11. Strathmann, H. - Pure Appl. Chem., 46, p. 213 (1976).

12. Edgar, K. J. - Cellulose, 14, p. 49 (2007).
13. Liao, Z.; Klein, E.; Poh, C. K.; Huang, Z.; Lu, J.; Hardy, P. A. \& Gao, D. - J. Membrane Sci., 256, p. 176 (2005).

14. Edgar, K. J.; Buchanam, C. M.; Debenham, J. S.; Rundquist, P. A.; Seiler, B. D.; Shelton, M. C. \& Tindall, D. - Prog. Pol. Deg., 26, p. 1605 (2001).

15. Sridhar, S.; Smitha, B. \& Aminabhavi, T. M. - Separat. Purif. Rev., 36, p. 113 (2007).

16. Kobayashi, Y.; Ishizaka, T. \& Kurokawa, Y. - J. Mater. Sci., 40, p. 263 (2005).

17. Cerqueira, D. A.; Valente, A. J. M.; Filho, G. R. \& Burrows, H. D. - Carbohyd. Polym., 78, p. 402 (2009).

18. Douglas, P. \& Eaton, K. - Sensor Actuator B Chem., 82, p. 200 (2002).

19. Tapia, M. J.; Valente, A. J. M.; Burrows, H. D.; Calderon, V.; Garcia, F. \& Garcia, J. M. - Eur. Polym. J., 43, p. 3838 (2007).

20. Valente, A. J. M.; Jiménez, A.; Simões, A. C.; Burrows, H. D.; Polishchuk, A. Y. \& Lobo, V. M. M. - Eur. Polym. J., 43, p. 2433 (2007).

21. Planes, J.; Wolter, A.; Cheguettine, Y.; Pron, A.; Genoud, F. \& Nechtschein, M. - Phys. Rev. B, 58, p. 7774 (1998).

22. Neves, S. \& Paoli, M. A. - Synth. Metals, 96, p. 49 (1998).

23. Paoli, M. A.; Duek, E. R. \& Rodrigues, M. A. - Synth. Met. 41, p. 973 (1991).

24. Lindfors, T.; Sjoberg, P.; Bobacka, J.; Lewenstam, A. \& Ivaska, A. - Anal. Chim. Acta, 385, p. 163 (1999).

25. Sassi, J. F. \& Chanzy, H. - Cellulose, 2, p. 111 (1995).

26. Samios, E.; Dart, R. K. \& Dawkins, J. V. - Polymer, 38, p. 3045 (1997).

27. Heinze, T. \& Liebert, T. - Macromol. Symp., 208, p. 167 (2004).

28. Cerqueira, D. A.; Rodrigues Filho, G. \& Meireles, C. S. - Carbohyd. Polym. 69, p. 579 (2007).

29. Rodrigues Filho, G.; Cruz, S. F.; Pasquini, D.; Cerqueira, D. A.; Prado, V. D. \& Assunção, R. M. N. - J. Memb. Sci., 177, p. 225 (2000).

30. ASTM INTERNATIONAL - "ASTM D871-96", Pennsylvania (2004).

Enviado: 28/10/09

Reenviado: 11/12/09

Aceito: $22 / 12 / 09$

DOI: $10.1590 / \mathrm{S} 0104-14282010005000017$ 